\title{
Non-Markovian Dynamics of an Open Two-Level System with Amplitude-Phase Damping
}

\author{
Ning Tang, Guoyou Wang, Zilong Fan, Haosheng Zeng* \\ Key Laboratory of Low-Dimensional Quantum Structures and Quantum Control of Ministry of \\ Education, Department of Physics, Hunan Normal University, Changsha, China \\ Email: "hszeng@hunnu.edu.cn
}

Received February 6, 2013; revised March 10, 2013; accepted March 19, 2013

\begin{abstract}
By use of the measure, the backflow of information presented recently, we study the non-Markovianity of the dynamics for a two-level system interacting with a zero-temperature structured environment via amplitude-phase coupling. In the limit of weak coupling between the system and its reservoir, the time-local non-Markovian master equation for the reduced state of the system is derived. Under the secular approximation, the exact analytic solution is obtained. Numerical simulations show that the amplitude and phase dampings can produce destructive interference to the backflow of information, leading to the weaker non-Markovianity of the compound dynamics compared with the dynamics of a single amplitude or phase damping model. We also study the characteristics of the initial-state pairs that maximize the backflow of information.
\end{abstract}

Keywords: Non-Markovianity; Amplitude-Phase Dampings; Destructive Interference

\section{Introduction}

The evolution of open quantum systems can be divided into two basic types, i.e., Markovian and non-Markovian processes. For memoryless Markovian processes, the environment acts as a sink and the information that the system released into the environment during their interaction no longer reflows to the system. However, this is not the case in the non-Markovian processes, where the lost information will return to the system at a later time, so that the later evolution of the system is affected by its past history, i.e., which appears memory effect.

Although almost all early works are devoted to the study of Markovian processes [1], people recently found that Many relevant physical systems, such as the quantum optical system, quantum dot [2], superconductor system [3], quantum chemistry [4] and biological system [5] etc. can not be described simply by Markovian dynamics. Quantum non-Markovian processes can lead to distinctly different effects on decoherence and disentanglement $[6$, 7] of open systems compared with Markovian processes, which are important both for the enriching of the basic theory of quantum mechanics and for some practical applications, such as the quantum metrology [8] and quantum key distribution [9]. Because of these distinct properties and extensive applications, more and more attentions and interest have been devoted to the study of

${ }^{*}$ Corresponding author.
non-Markovian processes of open systems, including the measures of non-Markovianity [10-17], the positivity [18, 19], and some other dynamical properties [20-26] of nonMarkovian processes. Experimentally, the simulation [27-30] of non-Markovian environment has been realized.

The study of non-Markovian dynamics of open quantum systems is typically very involved and often requires some assumptions or approximations. In the previous researches of non-Markovian dynamics, only a single coupling way (amplitude-damping or dephase interaction) is assumed. Further, the rotating wave approximation, that is, neglecting the counter-rotating terms in the system-reservoir interaction Hamiltonian, is employed. These assumptions and approximations limit the serviceable range of the model. In this paper, we consider a complex model which simultaneously consists of amplitude and phase dampings to the environment. We also reserve the counterrotating terms in the system-reservoir interaction Hamiltonian. Our motivation is to compare the nonMarkovian features of the dynamics for amplitude and phase dampings, and to observe the interference between the two non-Markovian dynamics induced by the two damping ways.

The article is organized as follows. In Section 2, we introduce the microscopic Hamiltonian model and derive the non-Markovian time-local master equation for a twolevel system weakly coupled to a vacuum reservoir. In 
Section 3, we solve the master equation under the socalled secular approximation and present the analytic expression for the calculation of non-Markovianity based on the measure proposed by Breuer, Laine and Piilo (BLP) [10] recently. In Section 4, we choose the Lorentzian spectra reservoir as an exemplary example and simulate numerically the non-Markovianity of the system dynamics. The non-Markovian effects produced by amplitude noise, phase noise and their combination are investigated. And finally the conclusion is arranged in Section 5 .

\section{Microscopic Model}

Consider a two-level atom with Bohr frequency $\omega_{0}$ interacting with a zero-temperature bosonic reservoir modeled by an infinite chain of quantum harmonic oscillators. The total Hamiltonian for this system in the Schrodinger picture is given by.

$$
\begin{aligned}
H= & \frac{1}{2} \omega_{0} \sigma_{z}+\sum_{k} \omega_{k} b_{k}^{+} b_{k}+\sum_{k} \beta g_{k} \sigma_{z}\left(b_{k}+b_{k}^{+}\right) \\
& +\sum_{k} \alpha g_{k}\left(\sigma_{+}+\sigma_{-}\right)\left(b_{k}+b_{k}^{+}\right) .
\end{aligned}
$$

where $\sigma_{z}$ and $\sigma_{ \pm}$are the Pauli and inversion operators of the atom, $\omega_{k}, b_{k}$ and $b_{k}^{+}$are respectively the frequency, annihilation and creation operators for the $k$-th harmonic oscillator of the reservoir. The atom couples to its environment via both amplitude and phase interactions, $g_{k}$ is the coupling strength which is assumed to be real for simplicity. The parameters $\alpha$ and $\beta$ describe the relative strengthes of the two couplings which satisfy $\alpha^{2}+\beta^{2}=1$. Note that we include the counter-rotating terms, $\sigma_{+} b_{k}^{+}$and $\sigma_{-} b_{k}$, in the interaction Hamiltonian.

The time-convolutionless (TCL) projection operator technique [1] is most effective in dealing with the dynamics of open quantum systems. In the limit of weak coupling between the system and its environment, by expanding the TCL generator to the second order with respect to coupling strength, the non-Markovian master equation describing the evolution of the reduced system, in the interaction picture, can be written as

$$
\frac{\mathrm{d} \rho(t)}{\mathrm{d} t}=-i\left[H_{L S}(t), \rho(t)\right]+D[\rho(t)]+D^{\prime}[\rho(t)] \text {. }
$$

where

$$
H_{L S}(t)=\alpha^{2}\left[S_{-}(t) \sigma_{+} \sigma_{-}+S_{+}(t) \sigma_{-} \sigma_{+}\right],
$$

is the Lamb shift Hamiltonian which describes a small shift in the energy of the eigenstates of the two-level atom. In many theoretical researches [20], this term was neglected usually. But in this paper, we will take it into the consideration. The parameters $S_{+}$and $S_{-}$which corresponds to respectively the Lamb shifts of levels $|0\rangle$ and $|1\rangle$ may be written as

$$
S_{\xi}(t)=-\int_{0}^{t} \mathrm{~d} \tau \int \mathrm{d} \omega J(\omega) \sin \left[\left(\omega_{0}+\xi \omega\right) \tau\right],
$$

with $\xi=\{+1,-1\}$ and $J(\omega)=\sum_{k}\left|g_{k}\right|^{2} \delta\left(\omega-\omega_{k}\right)$ the spectral distribution of the environment.

The dissipator $D[\rho(t)]$ that describes the secular motion of the system has the form

$$
\begin{aligned}
D[\rho(t)]= & \alpha^{2} \Gamma_{-}(t)\left\{\sigma_{-} \rho(t) \sigma_{+}-\frac{1}{2}\left\{\sigma_{+} \sigma_{-}, \rho(t)\right\}\right\} \\
& +\alpha^{2} \Gamma_{+}(t)\left\{\sigma_{+} \rho(t) \sigma_{-}-\frac{1}{2}\left\{\sigma_{-} \sigma_{+}, \rho(t)\right\}\right\} \\
& +\beta^{2} \Gamma_{0}(t)\left\{\sigma_{z} \rho(t) \sigma_{z}-\rho(t)\right\},
\end{aligned}
$$

where the first term describes the dissipation of the atom to its environment with time-dependent decay rate $\Gamma_{-}(t)$, and the second term denotes the heating with a rate $\Gamma_{+}(t)$. The last term describes the purely dephasing with a rate $\Gamma_{0}(t)$. These time-dependent rates can be written as,

$$
\Gamma_{\varsigma}(t)=2 \int_{0}^{t} \mathrm{~d} \tau \int \mathrm{d} \omega J(\omega) \cos \left[\left(\omega+\varsigma \omega_{0}\right) \tau\right],
$$

with $\varsigma=\{-1,+1,0\}$.

The dissipator $D^{\prime}[\rho(t)]$ represents the contribution of the so-called nonsecular terms, that is, terms oscillating rapidly with Bohr frequency $\omega_{0}$,

$$
\begin{aligned}
D^{\prime} & {[\rho(t)] } \\
= & \alpha^{2}[u(t)-i v(t)] \sigma_{-} \rho(t) \sigma_{-} \\
& -\alpha \beta\left[\lambda_{-}(t)+i \theta_{-}(t)\right] \sigma_{+} \sigma_{z} \rho(t) \\
& -\alpha \beta\left[\lambda_{+}(t)-i \theta_{+}(t)\right] \sigma_{-} \sigma_{z} \rho(t) \\
& -\alpha \beta\left[\eta_{-}(t)+i \vartheta_{-}(t)\right] \sigma_{z} \sigma_{+} \rho(t) \\
& -\alpha \beta\left[\eta_{+}(t)-i \vartheta_{+}(t)\right] \sigma_{z} \sigma_{-} \rho(t) \\
& +\alpha \beta\left[\lambda_{+}(t)+\eta_{-}(t)+i \theta_{+}(t)+i \vartheta_{-}(t)\right] \sigma_{+} \rho(t) \sigma_{z} \\
& +\alpha \beta\left[\lambda_{-}(t)+\eta_{+}(t)-i \theta_{-}(t)-i \vartheta_{+}(t)\right] \sigma_{-} \rho(t) \sigma_{z}+h . c .,
\end{aligned}
$$

here h.c. denotes the Hermitian conjugation, and the time-dependent coefficients are defined as

$$
\begin{gathered}
u(t)=2 \int_{0}^{t} \mathrm{~d} \tau \int \mathrm{d} \omega J(\omega) \cos [\omega(t-\tau)] \cos \left[\omega_{0}(t+\tau)\right] \\
v(t)=2 \int_{0}^{t} \mathrm{~d} \tau \int \mathrm{d} \omega J(\omega) \cos [\omega(t-\tau)] \sin \left[\omega_{0}(t+\tau)\right] \\
\lambda_{\xi}(t)=\int_{0}^{t} \mathrm{~d} \tau \int \mathrm{d} \omega J(\omega) \cos \left(\omega_{0} t+\xi \omega \tau\right) \\
\eta_{\xi}(t)=\int_{0}^{t} \mathrm{~d} \tau \int \mathrm{d} \omega J(\omega) \cos \left[\omega_{0} \tau-\xi \omega(\tau-t)\right]
\end{gathered}
$$




$$
\begin{array}{r}
\theta_{\xi}(t)=\int_{0}^{t} \mathrm{~d} \tau \int \mathrm{d} \omega J(\omega) \sin \left(\omega_{0} t+\xi \omega \tau\right), \\
\vartheta_{\xi}(t)=\int_{0}^{t} \mathrm{~d} \tau \int \mathrm{d} \omega J(\omega) \sin \left[\omega_{0} \tau-\xi \omega(\tau-t)\right] .
\end{array}
$$

\section{Solution and Non-Markovian Measure}

We now consider the case where the nonsecular term $D^{\prime}[\rho(t)]$ can be neglected. Just as pointed out by

Maniscalco [31], this kind of secular approximation that used after tracing over the bath degrees of freedom is different from the rotating wave approximation before the tracing. It is a more precise approximation that consists in an average over rapidly oscillating terms, but does not wash out the effect of the counter-rotating terms present in the coupling Hamiltonian. Under the secular approximation, the master Equation (2) has the Lindbladlike form with time-dependant decay rates. It is straightforward and easy to show that the corresponding Bloch equation may be written as

$$
\begin{gathered}
b_{x}=-\frac{1}{2}\left(\alpha^{2} \Gamma_{-}+\alpha^{2} \Gamma_{+}+4 \beta^{2} \Gamma_{0}\right) b_{x}+\alpha^{2}\left(S_{+}-S_{-}\right) b_{y}, \\
b_{y}=-\frac{1}{2}\left(\alpha^{2} \Gamma_{-}+\alpha^{2} \Gamma_{+}+4 \beta^{2} \Gamma_{0}\right) b_{y}-\alpha^{2}\left(S_{+}-S_{-}\right) b_{x}, \\
b_{z}=-\alpha^{2}\left(\Gamma_{-}+\Gamma_{+}\right) b_{z}+\alpha^{2}\left(\Gamma_{+}-\Gamma_{-}\right),
\end{gathered}
$$

where the three components of the Bloch vector are defined as $b_{j}(t)=\operatorname{tr}\left[\rho(t) \sigma_{j}\right]$ with $j=x, y, z$ and $\sigma_{j}$ the Pauli operators. For compactness we omit the argument of all the time-dependent coefficients.

Employing the method proposed by Hall [32], the corresponding Bloch Equations (14)-(16) can be solved exactly which gives

$$
\begin{aligned}
& b_{x}(t)=\mathrm{e}^{-\Theta(t)}\left[b_{x}(0) \cos \delta(t)-b_{y}(0) \sin \delta(t)\right], \\
& b_{y}(t)=\mathrm{e}^{-\Theta(t)}\left[b_{x}(0) \sin \delta(t)+b_{y}(0) \cos \delta(t)\right], \\
& b_{z}(t)=\mathrm{e}^{-\Lambda(t)} b_{z}(0)+\mathrm{e}^{-\Lambda(t)} \int_{0}^{t} \mathrm{dse}^{\Lambda(s)} \alpha^{2}\left[\Gamma_{+}(s)-\Gamma_{-}(s)\right],
\end{aligned}
$$

with

$$
\begin{gathered}
\Theta(t)=\frac{1}{2} \int_{0}^{t} \mathrm{~d} s\left[\alpha^{2} \Gamma_{-}(s)+\alpha^{2} \Gamma_{+}(s)+4 \beta^{2} \Gamma_{0}(s)\right], \\
\Lambda(t)=\int_{0}^{t} \mathrm{~d} s \alpha^{2}\left[\Gamma_{-}(s)+\Gamma_{+}(s)\right],
\end{gathered}
$$

and

$$
\delta(t)=\int_{0}^{t} \mathrm{~d} s \alpha^{2}\left[S_{-}(s)-S_{+}(s)\right] .
$$

In this paper, we employ the (BLP) measure [10] to describe the non-Markovianity of the considered system. Note that Markovian processes always tend to continu- ously reduce the trace distance between any two states of a quantum system, thus an increase of the trace distance during any time interval implies the emergence of nonMarkovianity. BLP further linked the change of the trace distance to the flow of information between the system and its environment, and concluded that the backflow of information from environment to the system is the key feature of a non-Markovian dynamics. In quantum information science, the trace distance for quantum states $\rho_{1}$ and $\rho_{2}$ is defined as [33]

$$
D\left(\rho_{1}, \rho_{2}\right)=\frac{1}{2} \operatorname{tr}\left|\rho_{1}-\rho_{2}\right|,
$$

with trace norm defined as $|A|=\sqrt{A^{+} A}$. For a given pair of initial states $\rho_{1,2}(0)$ of the system, the change of the dynamical trace-distance can be described by its time derivative

$$
\sigma\left(t, \rho_{1,2}(0)\right)=\frac{\mathrm{d}}{\mathrm{d} t} D\left(\rho_{1}(t), \rho_{2}(t)\right),
$$

where $\rho_{1,2}(t)$ are the dynamical states of the system with the initial states $\rho_{1,2}(0)$. For Markovian processes, the monotonically reduction of the trace distance implies $\sigma\left(t, \rho_{1,2}(0)\right) \leq 0$ for any initial states $\rho_{1,2}(0)$ and at any time $t$. If there exists a pair of initial states of the system such that for some evolutional time $t$,

$\sigma\left(t, \rho_{1,2}(0)\right)>0$, then the information takes backflow from environment to the system, and the process is nonMarkovian. In order to describe the degrees of non-Markovianity of the whole dynamical process, the quantity,

$$
=\max _{\rho_{1,2}(0)} \int_{\sigma>0} \mathrm{~d} t \sigma\left(t, \rho_{1,2}(0)\right),
$$

is introduced. Where the time integration is extended over all intervals in which $\sigma$ is positive, and the maximum is taken over all initial-state pairs of the system. For any Markovian process, $=0$. The larger the quantity is, the higher the non-Markovianity of the process is.

For our considered open system and by use of the solution of Equations (17)-(22), Equations (24) becomes

$$
\begin{aligned}
\sigma= & -\frac{1}{4} G(t)\left\{\mathrm{e}^{-2 \Theta(t)}\left(\alpha^{2} \Gamma_{-}+\alpha^{2} \Gamma_{+}+4 \beta^{2} \Gamma_{0}\right)\right. \\
& \left.\times\left[\left(\Delta b_{x}\right)^{2}+\left(\Delta b_{y}\right)^{2}\right]+2 \mathrm{e}^{-2 \Lambda(t)} \alpha^{2}\left(\Gamma_{-}+\Gamma_{+}\right)\left(\Delta b_{z}\right)^{2}\right\},
\end{aligned}
$$

where

$$
G(t)=\left\{\mathrm{e}^{-2 \Theta(t)}\left[\left(\Delta b_{x}\right)^{2}+\left(\Delta b_{y}\right)^{2}\right]+\mathrm{e}^{-2 \Lambda(t)}\left(\Delta b_{z}\right)^{2}\right\}^{-1 / 2} \text { and }
$$

$\Delta b_{j}=b_{1 j}(0)-b_{2 j}(0)$ is the difference between the two Bloch components that correspond to the initial states $\rho_{1,2}(0)$ 


\section{Numerical Simulation}

In order to demonstrate quantitatively the non-Markovian characteristics of the system dynamics, we specify our study to a particular reservoir spectra, Lorentzian spectra,

$$
J(\omega)=\frac{\gamma_{0} \lambda^{2}}{2 \pi\left[\left(\omega_{0}-\omega-\Delta\right)^{2}+\lambda^{2}\right]},
$$

which describes the interaction of an atom with an imperfect cavity and is widely used in literatures. Where $\omega_{0}$ denotes the transition frequency of the atom,

$\Delta=\omega_{0}-\omega$ is the frequency detuning between the atom and the cavity mode. $\lambda$ is the width of Lorentzian distribution, which is connected to the reservoir correlation time $\tau_{R}=\lambda^{-1}$. The parameter $\gamma_{0}$ can be regarded as the decay rate for the excited atom in the Markovian limit of flat spectrum which is related to the relaxation time $\tau_{S}=\gamma_{0}^{-1}$. For the Lorentzian spectra, all the time-dependent coefficients can be calculated analyticcally,

$$
\begin{gathered}
S_{\xi}(t)=\xi \frac{\gamma_{0} \lambda}{2\left(\lambda^{2}+\Delta_{\xi}^{2}\right)}\left\{\mathrm{e}^{-\lambda t}\left[\lambda \sin \left(\Delta_{\xi} t\right)+\Delta_{\xi} \cos \left(\Delta_{\xi} t\right)\right]-\Delta_{\xi}\right\}, \\
\Gamma_{\varsigma}(t)=\frac{\gamma_{0} \lambda}{\lambda^{2}+\Delta_{\varsigma}^{2}}\left\{\mathrm{e}^{-\lambda t}\left[\Delta_{\varsigma} \sin \left(\Delta_{\varsigma} t\right)-\lambda \cos \left(\Delta_{\varsigma} t\right)\right]+\lambda\right\},
\end{gathered}
$$

with $\xi=\{-1,+1\}, \varsigma=\{-1,+1,0\}, \Delta_{-}=\omega_{0}-\omega=\Delta$, $\Delta_{+}=\omega_{0}+\omega=2 \omega_{0}-\Delta$, and $\Delta_{0}=\omega_{c}=\omega_{0}-\Delta$.

In Figure 1, we plot the non-Markovianity as a function of the weight factor $\alpha$ and the dimensionless de-

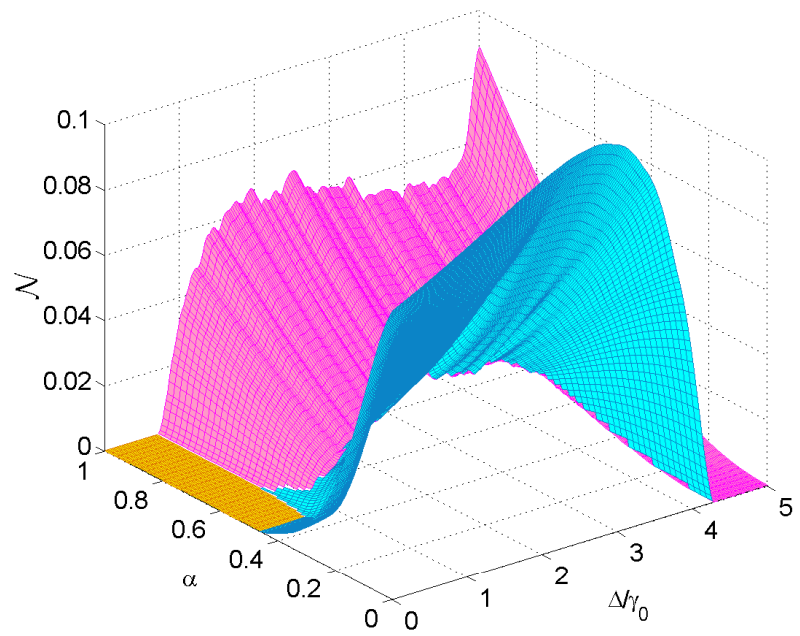

Figure 1. (Color online) Non-Markovianity

as a function of the weight factor $\alpha$ and the dimensionless detuning $\Delta / \gamma_{0}$ for $\omega_{0}=5 \gamma_{0}$ and $\lambda=0.2 \gamma_{0}$. Except for the orange domain in which $=0$, all the other parameter domains have nonzero non-Markovianity. tuning $\Delta / \gamma_{0}$, where the parameters are taken as $\omega_{0}=5 \gamma_{0}$ and $\lambda=0.2 \gamma_{0}$. It is shown that except for the orange domain in which $\quad=0$, all the other parameter domains (i.e., the red and blue domains) have nonzero non-Markovianity. $\alpha=1$ denotes the amplitude damping noise in which $=0$ for a small section of the beginning part of $\Delta / \gamma_{0}$, and then rises with the increase of the detuning, but the change is not monotonous. The changing regulation is very similar to that of the damped Jaynes-Cummings model case [10]. But here the effect of the counter-rotating terms is considered which induces some tiny oscillations of the non-Markovianity (see the dashed line in Figure 2) compared with the damped Janes-Cummings model case. $\alpha=0$ denotes the purely dephasing noise in which increases firstly from nonzero and then decreases when the detuning increases. Interestingly, for the detunings up to $\Delta / \omega_{0}=0.79$ which indeed covers all the reasonable detunings of practical experiments, the non-Markovianity induced by the purely dephasing noise is apparently lager than the one induced by purely amplitude-damping noise (see Figure 2), that is, in the same coupling strength, dephasing is more preferable to induce the backflow of information than dissipation. This is in accordance with the prediction [34] that the main contribution to the non-Markovianity stems from the evolution of the system coherence, because dephase interaction may be more preferable to induce the time evolution of the off-diagonal elements of the system density matrix than dissipation. Further we find that the superimposed interaction of phase and amplitude damplings $(0<\alpha<1)$ can suppress the backflow of information, leading to smaller non-Markovianity for measure . It means that in

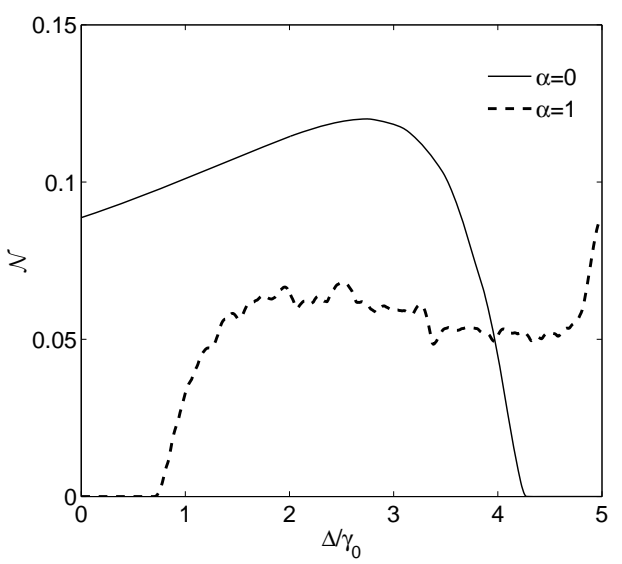

Figure 2. Non-Markovianity as a function of the dimensionless detuning $\Delta / \gamma_{0}$ for amplitude and phasing dampings for $\omega_{0}=5 \gamma_{0}$ and $\lambda=0.2 \gamma_{0}$. For the detunings up to $\Delta / \omega_{0}=3.97 \gamma_{0} / 5 \gamma_{0}=0.79$, the non-Markovianity

induced by the phase damping is lager than that induced by amplitude damping. 
practice we may, not only by the engineering of environmental structure but also by the adjusting of the coupling ways between the system and its environment, manipulate the non-Markovian dynamics of open quantum systems.

In Figure 3, we plot the measure as a function of the weight factor $\alpha$ and the dimensionless decay rate $\gamma_{0} / \lambda$, where the parameters is chosen as $\omega_{0}=25 \lambda$ and $\Delta=5 \lambda$. We find that for a given weight factor $\alpha$, the measure increases monotonously with decay rate $\gamma_{0} / \lambda$. This may be understood easily: The parameter $\lambda$ is the inverse of the reservoir correlation time. The decrease of $\lambda$ indicates an increase of the reservoir correlation time. The parameter $\gamma_{0}$ is the inverse of the system relaxation time. Decreasing the system relaxation time is equivalent to increasing the reservoir correlation time. Both the two changes can enhance the non-Markovianity of the system dynamics [35]. Note that for $\alpha=1$, the result is similar to the one of damped Jaynes-cummings model with resonant interaction [36]. Further we also find that the dephase noise $(\alpha=0)$ can give rise to much more non-Markovianity than amplitude-damping noise $(\alpha=1)$, and their superposition $(0<\alpha<1)$ can reduce the value of the measure. These results once again demonstrate the dominating rules of the quantum coherence to the non-Markovianity of open quantum system and the effect of destructive interference between amplitude and phase dampings.

We also investigate the problem of what initial state pairs can maximize the measure . Interestingly, in our numerical simulations, we find that there exist only two types of such initial state pairs. One pair is $|0\rangle$ and |1) which in Figures $\mathbf{1}$ and $\mathbf{3}$ applies to the red parame-

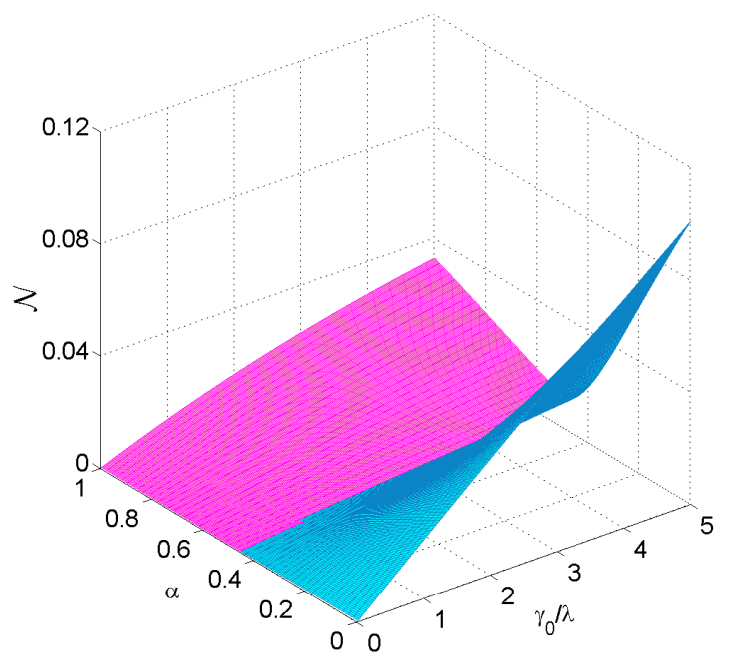

Figure 3. (Color online) Non-Markovianity as a function of the weight factor and the dimensionless decay rate $\gamma_{0} / \lambda$ for $\omega_{0}=25 \lambda$ and $\Delta=5 \lambda$. ter domain, the other pair is $|+\rangle=\frac{1}{\sqrt{2}}(|0\rangle+|1\rangle)$ and $|-\rangle=\frac{1}{\sqrt{2}}(|0\rangle-|1\rangle)$ which applies to the blue parameter domain. Both the initial state pairs have the largest trace distance. For the orange parameter domain in Figure 1, the measure $=0$. Note that the initial state pair $\{|0\rangle,|1\rangle\}$ agree with the result [10] for the damped Jaynes-Cummings model, though here we have considered the effect of counter-rotating terms in the interaction Hamiltonian. However, the initial state pair $\{|+\rangle,|-\rangle\}$ does not coincide with the result $\{|+\rangle,|0\rangle\}$ of reference [36]. The former is composed by two orthogonal states with maximal coherence. While the later contains the ground state and a maximal coherence state which have no the largest trace distance.

\section{Conclusions}

In conclusion, we have studied the non-Markovianity of the dynamics for a two-level system interacting with a zero-temperature structured environment via amplitudephase damping. In the limit of weak coupling between the system and the reservoir, we have derived the timelocal non-Markovian master equation for the reduced state of the system. The exact analytic solution under the secular approximation has been gained and the nonMarkovian properties for the system dynamics based on the BLP measure have been studied. We found, in the same coupling strength between the system and its environment, that the dephasing interaction is more beneficial to induce the backflow of information from the environment to the system compared with amplitude-damping model, leading to more stronger non-Markovianity for the system dynamics. This result further proves the previous viewpoint that the main contribution to the non-Markovianity stems from the evolution of the system coherence. We also found that the information backflows induced by amplitude and phase damplings can take destructive interference so as to suppress the non-Markovianity of the system dynamics.

For a given system-reservoir coupling model (a fixed $\alpha$ ), the non-Markovian measure increases monotonously with $\gamma_{0} / \lambda$, but it is not monotonous with dimensionless detuning $\Delta / \gamma_{0}$. The physical explanation for the former case is clear, but for the later case the reason is not clear yet and requires further studying.

In the definition of BLP non-Markovianity measure, a maximization over all possible initial state pairs is involved. This maximization process usually requires to spend much time or effort on the practical calculations. In our numerical simulations, we found that there are only two pairs of such initial states. One pair was found previously but another pair is new. For various different 
kinds of dynamical models, looking for the characteristics of such initial state pairs is perhaps a problem worthy of consideration [37,38].

The measure of non-Markovianity is a fundamental problem in the study of open quantum system dynamics. In the numerical simulations, we have only considered the Lorentzian environment. Actually, our analytic results also adapt to other structured environments, such as the Ohmic reservoir, the photonic band-gap material [39], etc. By properly engineering the structure of the environment, one can selectively alter the non-Markovian dynamical property of the open quantum system, so as to effectively control the evolution of some interesting physical quantities, such as the quantum coherence, quan tum entanglement and discord. Therefore, our work will also be helpful for the researches of the related problems.

\section{Acknowledgements}

This work is supported by the National Natural Science Foundation of China (Grant Nos. 11275064, 11075050), Specialized Research Fund for the Doctoral Program of Higher Education (Grant No. 20124306110003) the Construct Program of the National Key Discipline, the Program for Changjiang Scholars and Innovative Research Team in University under Grant No. IRT0964, and Hunan Provincial Natural Science Foundation under Grant No. 11JJ7001.

\section{REFERENCES}

[1] H. P. Breuer and F. Petruccione, "The Theory of Open Quantum Systems," Oxford University Press, Oxford, 2007. doi:10.1093/acprof:oso/9780199213900.001.0001

[2] Y. Kubota and K. Nobusada, "Applicability of Site-Basis Time-Evolution Equation for Thermalization of Exciton States in a Quantum Dot Array," Journal of the Physical Society of Japan, Vol. 78, No. 11, 2009, Article ID: 114603. doi:10.1143/JPSJ.78.114603

[3] Y. H. Ji and J. J. Hu, "Entanglement and Decoherence of Coupled Superconductor Qubits in a Non-Markovian Environments," Chinese Physics B, Vol. 19, No. 6, 2010, Article ID: 060304. doi:10.1088/1674-1056/19/6/060304

[4] J. Shao, "Decoupling Quantum Dissipation Interaction via Stochastic Fields," Journal of Chemical Physics, Vol. 120, No. 11, 2004, p. 5053. doi:10.1063/1.1647528

[5] A. W. Chin, A. Datta, F. Caruso, S. F. Huelga and M. B. Plenio, "Noise-Assisted Energy Transfer in Quantum Networks and Light-Harvesting Complexes," New Journal of Physics, Vol. 12, No. 6, 2010, Article ID: 065002. doi:10.1088/1367-2630/12/6/065002

[6] A. G. Dijkstra and Y. Tanimura, "Non-Markovian Entanglement Dynamics in the Presence of System-Bath Coherence," Physical Review Letters, Vol. 104, No. 25, 2010, Article ID: 250401. doi:10.1103/PhysRevLett.104.250401

[7] B. Bellomo, R. Lo Franco and G. Compagno, "Non-Mar- kovian Effects on the Dynamics of Entanglement," Physical Review Letters, Vol. 99, No. 16, 2007, Article ID: 160502. doi:10.1103/PhysRevLett.99.160502

[8] A. W. Chin, S. F. Huelga and M. B. Plenio, "Quantum Metrology in Non-Markovian Environments," Physical Review Letters, Vol. 109, No. 23, 2012, Article ID: 233601. doi:10.1103/PhysRevLett.109.233601

[9] R. Vasile, S. Olivares, M. G. A. Paris and S. Maniscalco, "Continuous-Variable Quantum Key Distribution in NonMarkovian Channels," Physical Review A, Vol. 83, No. 4, 2011, Article ID: 042321 doi:10.1103/PhysRevA.83.042321

[10] H. P. Breuer, E. M. Laine and J. Piilo, "Measure for the Degree of Non-Markovian Behavior of Quantum Processes in Open Systems," Physical Review Letters, Vol. 103, No. 21, 2009, Article ID: 210401. doi:10.1103/PhysRevLett.103.210401

[11] A. Rivas, S. F. Huelga and M. B. Plenio, "Entanglement and Non-Markovianity of Quantum Evolutions," Physical Review Letters, Vol. 105, No. 5, 2010, Article ID: 050403. doi:10.1103/PhysRevLett.105.050403

[12] M. M. Wolf, J. Eisert, T. S. Cubitt and J. I. Cirac, "Assessing Non-Markovian Quantum Dynamics," Physical Review Letters, Vol. 101, No. 15, 2008, Article ID: 150402. doi:10.1103/PhysRevLett.101.150402

[13] A. R. Usha Devi, A. K. Rajagopal and Sudha, "OpenSystem Quantum Dynamics with Correlated Initial States, Not Completely Positive Maps, and Non-Markovianity," Physical Review A, Vol. 83, No. 2, 2011, Article ID: 022109. doi:10.1103/PhysRevA.83.022109

[14] X. M. Lu, X. G. Wang and C. P. Sun, "Quantum Fisher Information Flow and Non-Markovian Processes of Open Systems," Physical Review A, Vol. 82, No. 4, 2010, Article ID: 042103. doi:10.1103/PhysRevA.82.042103

[15] S. Luo, S. Fu and H. Song, "Quantifying Non-Markovianity via Correlations," Physical Review A, Vol. 86, No. 4, 2012, Article ID: 044101. doi:10.1103/PhysRevA.86.044101

[16] D. Chruscinski, A. Kossakowski and A. Rivas, "Measures of Non-Markovianity: Divisibility versus Backflow of Information," Physical Review A, Vol. 83, No. 5, 2011, Article ID: 052128. doi:10.1103/PhysRevA.83.052128

[17] H. S. Zeng, N. Tang, Y. P. Zheng and G. Y. Wang, "Equivalence of the Measures of Non-Markovianity for Open Two-Level," Physical Review A, Vol. 84, No. 3, 2011 , Article ID: 032118. doi:10.1103/PhysRevA.84.032118

[18] A. Shabani and D. A. Lidar, "Vanishing Quantum Discord is Necessary and Sufficient for Completely Positive Maps," Physical Review Letters, Vol. 102, No. 10, 2009, Article ID: 100402. doi:10.1103/PhysRevLett.102.100402

[19] H. P. Breuer and B. Vacchini, "Structure of Completely Positive Quantum Master Equations with Memory Kernel," Physical Review E, Vol. 79, No. 4, 2009, Article ID: 041147. doi:10.1103/PhysRevE.79.041147

[20] P. Haikka and S. Maniscalco, "Non-Markovian Dynamics of a Damped Driven Two-State System," Physical Review A, Vol. 81, No. 5, 2010, Article ID: 052103. doi:10.1103/PhysRevA.81.052103 
[21] K. W. Chang and C. K. Law, "Non-Markovian Master Equation for a Damped Oscillator with Time-Varying Parameters," Physical Review A, Vol. 81, No. 5, 2010, Article ID: 052105. doi:10.1103/PhysRevA.81.052105

[22] W. J. Gu and G. Y. Li, "Non-Markovian Behavior for Spontaneous Decay of a V-Type Three-Level Atom with Quantum Interference," Physical Review A, Vol. 85, No. 1, 2012, Article ID: 014101. doi:10.1103/PhysRevA.85.014101

[23] D. Chruscinski, A. Kossakowski and S. Pascazio, "LongTime Memory in Non-Markovian Evolutions," Physical Review A, Vol. 81, No. 3, 2010, Article ID: 032101. doi:10.1103/PhysRevA.81.032101

[24] P. Haikka, J. D. Cresser and S. Maniscalco, "Comparing Different Non-Markovianity Measures in a Driven Qubit System," Physical Review A, Vol. 83, No. 1, 2011, Article ID: 012112. doi:10.1103/PhysRevA.83.012112

[25] X. Yin, J. Ma, X. Wang and F. Nori, "Spin Squeezing under Non-Markovian Channels by the Hierarchy Equation Method," Physical Review A, Vol. 86, No. 1, 2012, Article ID: 012308. doi:10.1103/PhysRevA.86.012308

[26] J. Li, G. McKeown, F. L. Semiao and M. Paternostro, "NonMarkovian Effects on the Nonlocality of a Qubit-Oscillator System," Physical Review A, Vol. 85, No. 2, 2012, Article ID: 022116. doi:10.1103/PhysRevA.85.022116

[27] J. S. Xu, C. F. Li, M. Gong, X. B. Zou, C. H. Shi, G. Chen and G. C. Guo, "Experimental Demonstration of Photonic Entanglement Collapse and Revival," Physical Review Letters, Vol. 104, No. 10, 2010, Article ID: 100502. doi:10.1103/PhysRevLett.104.100502

[28] J. S. Xu, C. F. Li, C. J. Zhang, X. Y. Xu, Y. S. Zhang and G. C. Guo, "Experimental Investigation of the Non-Markovian Dynamics of Classical and Quantum Correlations," Physical Review A, Vol. 82, No. 4, 2010, Article ID: 042328. doi:10.1103/PhysRevA.82.042328

[29] B. H. Liu, L. Li, Y. F. Huang, C. F. Li, G. C. Guo, E. M. Laine, H. P. Breuer and J. Piilo, "Experimental Control of the Transition from Markovian to Non-Markovian Dynamics of Open Quantum Systems," Nature Physics, Vol. 7, No. 12, 2011, pp. 931-934. doi:10.1038/nphys2085

[30] J. S. Tang, C. F. Li, Y. L. Li, X. B. Zou, G. C. Gou, H. P. Breuer, E. M. Laine and J. Piilo, "Measuring Non-Mar- kovianity of Processes with Controllable System-Environment Interaction," Europhysics Letters, Vol. 97, No. 1, 2012, Article ID: 10002. doi:10.1209/0295-5075/97/10002

[31] S. Maniscalco, J. Piilo, F. Intravaia, F. Petruccione and A. Messina, "Lindblad- and Non-Lindblad-Type Dynamics of a Quantum Brownian Particle," Physical Review A, Vol. 70, No. 3, 2004, Article ID: 032113. doi:10.1103/PhysRevA.70.032113

[32] M. J. W. Hall, "Complete Positivity for Time-Dependent Qubit Master Equations," Journal of Physics A: Mathematical and Theoretical, Vol. 41, No. 20, 2008, Article ID: 205302. doi:10.1088/1751-8113/41/20/205302

[33] M. A. Nielsen and I. L. Chuang, "Quantum Computation and Quantum Information," Cambridge University Press, Cambridge, 2000.

[34] G. Clos and H. P. Breuer, "Quantification of Memory Effects in the Spin-Boson Model," Physical Review A, Vol. 86, No. 1, 2012, Article ID: 012115. doi:10.1103/PhysRevA.86.012115

[35] J.-G. Li, J. Zou and B. Shao, "Non-Markovianity of the Damped Jaynes-Cummings Model with Detuning," Physical Review A, Vol. 81, No. 6, 2010, Article ID: 062124. doi:10.1103/PhysRevA.81.062124

[36] E. M. Laine, J. Piilo and H. P. Breuer, "Measure for the Non-Markovianity of Quantum Processes," Physical Review A, Vol. 81, No. 6, 2010, Article ID: 062115. doi:10.1103/PhysRevA.81.062115

[37] Z. Y. Xu, W. L. Yang and M. Feng, "Proposed Method for Direct Measurement of the Non-Markovian Character of the Qubits Coupled to Bosonic Reservoirs," Physical Review A, Vol. 81, No. 4, 2010, Article ID: 044105. doi:10.1103/PhysRevA.81.044105

[38] Z. He, J. Zou, L. Li and B. Shao, "Effective Method of Calculating the Non-Markovianity N for Single-Channel Open Systems," Physical Review A, Vol. 83, No. 1, 2011, Article ID: 012108. doi:10.1103/PhysRevA.83.012108

[39] M. Woldeyohannes and S. John, "Coherent Control of Spontaneous Emission near a Photonic Band Edge," Journal of Optics B: Quantum and Semiclassical Optics, Vol. 5, No. 2, 2003, p. 43. doi:10.1088/1464-4266/5/2/201 